\title{
THORAX
}

Editorials

\section{Liposomes for controlled delivery of drugs to the lung}

Liposomes are one of several drug delivery systems known pharmaceutically as vesicles. They can be prepared from phospholipids with or without cholesterol in a wide range of sizes, from $20 \mathrm{~nm}$ to $10 \mu \mathrm{m}$, and structurally have one or more lipid bilayers, separated by aqueous compartments, surrounding an aqueous core. Liposomes are capable of entrapping a wide range of materials; hydrophilic drugs are dissolved in the aqueous regions and hydrophobic drugs associated with the lipid bilayers. Liposomes have been administered to the lung both as a means of delivering phospholipids to the alveolar surface and to modulate the duration of activity or pulmonary absorption of pharmacologically active agents.

Liposomes seem particularly appropriate for delivery of drugs to the lungs as they can be prepared from materials endogenous to the lung as components of lung surfactant. Lung surfactant is a complex mixture, of which about $85 \%$ is phospholipid, mostly dipalmitoylphosphatidylcholine, with phosphatidylglycerol as the next most prevalent phospholipid. ${ }^{1}$ Surfactant also contains cholesterol ${ }^{1}$ and two groups of non-serum proteins that are considered important in the spreading, adsorption, and reutilisation of surfactant. ${ }^{2}$ The mechanisms for the clearance and reutilisation of lung surfactant are likely to be of major importance in determining the fate of liposomes deposited in the alveoli. Administration of radiolabelled liposomes to the respiratory tract of rodents has shown that liposomal phospholipid rapidly becomes associated with the lung parenchyma. ${ }^{34}$ Exogenous phospholipids enter the process for reutilising lung surfactant, being taken up by type II epithelial cells, and become incorporated into lamellar bodies. ${ }^{4}$ The rate and extent of pulmonary uptake of liposomes are a function of their composition, significantly faster rates occurring when liposomes contain phosphatidylglycerol. $^{3}$

A recent study of the acute effects of liposome inhalation by healthy volunteers indicated that small soya phosphatidylcholine liposomes were well tolerated, with no apparent changes in pulmonary function. ${ }^{5}$ Chronic administration of liposomes, however, may result in cumulative doses of phospholipid greater than the size of the lung surfactant pool. In clinical cases of phospholipidosis, occurring as an adverse reaction to amiodarone, concentrations of phospholipids in cell free bronchoalveolar lavage fluid remain normal but there is a large increase in the lavage cell population, predominantly of alveolar macrophages, and their phospholipid content. ${ }^{6}$ This suggests that macrophage activity may be an important clearance mechanism in response to chronic administration of liposomes.

Gamma scintigraphy has been used to study the deposition and clearance of radiolabelled liposomes in the human respiratory tract. These investigations have indicated that liposomes can be efficiently deposited in the lung, where they remain intact for prolonged periods. Whether they are deposited in the central or peripheral airways depends on the size of the droplets in the aerosols produced by nebulisers used for their delivery, rather than on properties of the vesicles themselves. The desired site of deposition within the lung will clearly be governed by the site of drug action. Rapid removal of particulates, including liposomes, by the mucociliary clearance process suggests that peripheral lung deposition will be required to achieve much longer drug action.

Multilamellar and small unilamellar liposomes with bilayers of dipalmitoylphosphatidylcholine, with a mean size of $2.9 \mu \mathrm{m}$ and $70 \mathrm{~nm}$ respectively, had their outer surface labelled with ${ }^{99 \mathrm{~m}} \mathrm{~T} \mathrm{c}$ before inhalation by healthy volunteers from a Hudson jet nebuliser. ${ }^{7}$ The distribution of activity within the lung depended on the size of droplets in the nebulised aerosol cloud (mass median aerodynamic diameter (MMAD) 3.2-3.7 $\mu \mathrm{m}$ ) rather than vesicle size or type. The rate of clearance of the two vesicle types was similar, with about $40 \%$ of deposited activity cleared within 20 hours by the mucociliary clearance process. The activity remaining in the lung after 20 hours probably represented vesicles that had been deposited in the alveoli.

Dipalmitoylphosphatidylcholine-cholesterol multilamellar liposomes (mean size $0.9 \mu \mathrm{m}$ ) with their aqueous phase labelled with ${ }^{99 \mathrm{~m}}$ Tc DTPA were atomised by a Respigard jet nebuliser (MMAD $1.2 \mu \mathrm{m})^{8}$ and inhaled by healthy volunteers. Such a small droplet size ensured peripheral lung deposition. Single photon emission computed tomography images one hour after inhalation showed that activity was widely distributed throughout the lungs. After 24 hours $45 \%$ of the initially deposited activity persisted within the lungs, representing the fraction of radiolabel remaining in intact liposomes in the alveolar regions, as free radiolabel was rapidly absorbed and cleared from the airways. When an equivalent dose of free ${ }^{99 \mathrm{~m}}$ Tc DTPA was inhaled as a solution, activity could not be detected in the pulmonary regions after six hours.

In the earliest studies of pulmonary administration of liposomes for therapeutic purposes liposomes were given as an exogenous lung surfactant in the treatment of the neonatal respiratory distress syndrome. ${ }^{9}$ Subsequently a range of liposome associated drugs have been administered to the lungs of humans and animals. These include cytotoxic drugs, ${ }^{10}$ antiasthma drugs, ${ }^{11}{ }^{12}$ antimicrobial compounds, ${ }^{13-15}$ and drugs delivered for a systemic action. ${ }^{15-17}$

When cytarabine was administered to the lungs of rabbits in free and liposomal form, the free drug was rapidly absorbed whereas the liposomal drug remained in the airways for prolonged periods, with little distribution to other tissues. Free cytarabine inhibited $\left[{ }^{14} \mathrm{C}\right]$ thymidine incorporation in the gastrointestinal tract, bone marrow, and lung, whereas liposomal drug effectively inhibited $\left[{ }^{14} \mathrm{C}\right]$ thymidine incorporation within lung tissue but with little effect in the gastrointestinal tract and bone marrow. ${ }^{10}$

Pulmonary administration of orciprenaline sulphate to guinea pigs produced bronchodilation of short duration accompanied by considerable tachycardia. Liposome for- 
mulations of that drug produced immediate bronchodilation after histamine induced bronchoconstriction, but with an appreciably prolonged duration of activity. ${ }^{11}$ Additionally, the cardiovascular side effects of orciprenaline were significantly reduced by liposomal incorporation.

Cytarabine and orciprenaline sulphate are hydrophilic drugs entrapped within the aqueous compartments of liposomes. Similar findings have been reported for hydrophobic drugs, such as atropine, associated with lipid bilayers. ${ }^{17}$ These studies show the potential of liposomes for prolonging the time that drugs associated with them are retained in the lung, resulting in localised high drug concentrations within the respiratory tract and decreased adverse effects at sites distant from the lung.

Studies of inhaled liposomal drug formulations in man are scarce. Liposomes containing the antiviral compound enviroxime have been inhaled by healthy volunteers after atomisation from a Puritan-Bennett jet nebuliser producing aerosols with mass median diameters of $2 \cdot 4-3 \cdot 1 \mu \mathrm{m} .{ }^{14}$ From 7 to $10 \mathrm{mg}$ of drug was deposited in the respiratory tract over one hour. Large amounts of drug were found in nasal washes one hour after inhalation. Enviroxime could not be detected in urine samples, and was detectable in only one of five blood samples. No side effects were observed and the authors concluded that such a liposome formulation had potential for delivering hydrophobic drugs for the treatment of respiratory disease. Nebulisation of free enviroxime is problematic owing to its poor solubility in water.

A Hudson jet nebuliser was used to produce aerosols (MMAD 2.6 $\mu \mathrm{m}$ ) from a dipalmitoylphosphatidylcholinecholesterol liposome formulation of sodium cromoglycate. This preparation when inhaled by healthy volunteers produced detectable plasma concentrations of drug up to 25 hours after inhalation. ${ }^{12}$ An equivalent $20 \mathrm{mg}$ dose of sodium cromoglycate inhaled as a nebulised solution produced peak plasma concentrations seven times greater than liposomal sodium cromoglycate, but the drug was not detectable in 25 hour plasma samples. Maximum, mean, and minimum steady state plasma concentrations likely to result from repeated administration of the liposomal formulation were calculated. These indicated that one dose a day would result in plasma concentrations of the drug that have been shown to be associated with a protective action in bronchial asthma.

The absorption half life of inhaled liposomal sodium cromoglycate was 57 hours, about three times faster than predicted from in vitro experiments. This suggests that diffusion of drug across liposome bilayers may not be the major determinant of drug release and subsequent plasma concentrations. Drug release may be enhanced by vesicle degradation at the alveolar surface or within alveolar macrophages, fusion of liposomes with epithelial cells, or fusion or phospholipid exchange (or both) with constituents of lung surfactant.

Currently liposome delivery to the lung in man is achieved through the use of nebulisers. ${ }^{781214}$ Formulation of liposomes for delivery from nebulisers is relatively simple. They can be prepared by conventional techniques and, except for removal of material not associated with the vesicles, usually require no further processing.

The effect of jet nebulisation on a range of liposome preparations of sodium cromoglycate has been investigated with a multistage liquid impinger. ${ }^{18}$ The size and size distribution of the droplets in the aerosols produced depended on the design of the nebuliser rather than any property of the liposomes themselves. The results suggest that the appropriate liposome formulation for delivery from such devices will be governed by the oil:water partition coefficient of the drug and its therapeutic dose.
Larger liposomes encapsulate more hydrophilic materials, but these vesicles are the least stable during atomisation. Reducing the size of liposomes by extrusion or sonication makes them more stable. Disruption of vesicles on atomisation is likely to be less critical for hydrophobic drugs, associated as they are with lipid bilayers.

The use of ultrasonic nebulisers for liposome delivery has been investigated. ${ }^{19}$ Small liposomes were stable during nebulisation, though dipalmitoylphosphatidylcholine vesicles of $500 \mathrm{~nm}$ or more increased in size within the nebuliser, suggesting fusion of vesicles, with the likely loss of entrapped hydrophilic materials.

In the future alternative devices may become available for delivery of liposomes to the lung. For instance, a recent United States patent ${ }^{20}$ described the use of a dry powder inhalation device for delivering vesicles loaded with spray dried drug. This approach to pulmonary delivery will rely on rehydration of vesicles in situ. The potential of metered dose inhalers has also been investigated. A solution phase system has been developed in which phospholipid and drug are dissolved in a chlorofluorocarbon based propellant blend. ${ }^{21}$ After inhalation of the emitted droplets it is postulated that rapid phospholipid hydration will result in spontaneous formation of vesicles. This approach has proved applicable for delivering systems containing small doses of salbutamol ${ }^{21}$ and would seem suitable for delivery of corticosteroids. Other approaches for metered dose inhaler systems include a two compartment system, in which a chlorofluorocarbon based solution containing phospholipid and drug is mixed with an aqueous phase on actuation, ${ }^{22}$ and a system in which preformed dehydrated liposomes are suspended in a chlorofluorocarbon blend with poor solubilising capacity for phospholipids. ${ }^{20}$ These alternative approaches are limited by the fact that smaller amounts of phospholipid or liposomes can be delivered than with nebulisers.

From a toxicological viewpoint, then, liposomes seem particularly appropriate for drug delivery to the lung. The limited data pertaining to their use in man and the results of animal studies indicate that encapsulation in liposomes can modulate the fate of inhaled drugs, increasing the residence time of drug in the lung. This may allow prolonged drug action within the respiratory tract or a prolonged presence in the circulation. By providing a controlled delivery of drug at the surface of the lung such systems may allow a decreased frequency of dosing, with a reduction in systemic side effects. How controllable drug release from a liposome system deposited in the lung will be has yet to be established, in healthy or diseased lungs. Generally, inclusion of cholesterol in liposomes increases in vivo stability, decreasing the rate of drug release. ${ }^{23}$ Studies of the fate of exogenous surfactant in the lung suggest that inclusion of phosphatidylglycerol aids spreading at the alveolar surface. ${ }^{3}$ Thus inclusion of phosphatidylglycerol in liposomes may potentially enhance drug release. If fusion of vesicles with the alveolar surface or lipid exchange between vesicles and surfactant (or both) is an important release mechanism, liposomes with more than one bilayer may be more appropriate than unilamellar liposomes for producing sustained drug release.

The ability of liposomes to encapsulate hydrophilic and hydrophobic drugs makes them potential carriers for many compounds. Drugs having small therapeutic doses will be obvious candidates, as concomitantly administered phospholipids can be minimised. Obvious drug candidates for future investigation are the $\beta_{2}$ agonists and corticosteroids such as beclomethasone. The sustained release of materials within the lung, together with the large absorptive alveolar surface, indicates that pulmonary delivery of liposomal drugs has potential not only for locally acting drugs but also 
for systemically active compounds, including peptides.

KMG TAYLOR

JM NEWTON

Department of Pharmaceutics,

School of Pharmacy,

University of London,

Correspondence to Dr Taylor

King RJ. Isolation and chemical composition of pulmonary surfactant. In: Robertson B, Van Golde LMG, Batenburg JJ, eds. Pulmonary surfactant. Amstedam: Elsevier, 1984:1-15.

2 Wright JR, Clements JA. Metabolism and turnover of lung surfactant. $A m$ Rev Respir Dis 1987;135:426-44.

3 Oyarzun MJ, Clements JA, Baritussio A. Ventilation enhances the pulmonary alveolar clearance of radioactive dipalmitoylphosphatidylcholine in liposomes. Am Rev Respir Dis 1980;121:709-21.

4 Morimoto Y, Adachi Y. Pulmonary uptake of liposomal phosphatidylcholine upon intratracheal administation to rats. Chem Pharmaceut Bull 1982; 30:2248-51.

5 Thomas DA, Myers MA, Wichert B, Schreier H, Gonzalez-Rothi RJ. Acute effects of liposome aerosol inhalation on pulmonary function in healthy human volunteers. Chest 1991;99:1268-70.

6 Martin WJ, Standing JE. Amiodarone pulmonary toxicity: biochemical evidence for a cellular phospholipidosis in the bronchoalveolar lavage of human subjects. J Pharmacol Exp Ther 1988;244:774-9.

7 Farr SJ, Kellaway IW, Parry-Jones DR, Woolfrey SG. 99m-Technetium as a marker of liposomal deposition and clearance in the human lung. Int $J$ Pharmaceut 1985;26:303-16.

8 Barker SA, Taylor KMG, Short MD. Gamma Scintigraphy of Inhaled Radioactive Liposomes [Abstract]. In: Proceedings of the 18th International Symposium on Controlled Release of Bioactive Materials 1991;18:289-90.

9 Ivey H, Roth S, Kattwinkel J. Nebulization of sonicated phospholipid for treatment of respiratory distress syndrome (RDS) of infancy. Paediatr Res 1977;11:573.

10 McCullough HN, Juliano RL. Organ-selective action of an antitumour drug: pharmacologic studies of liposome encapsulated Cytosine arabinoside

administered via the respiratory system of the rat. $J$ Natl Cancer Inst 1979;63:727-31.

11 McCalden TA, Abra RM, Mihalko PJ. Bronchodilator efficacy of liposome formulations of metaproteranol sulfate in the anesthetized guinea pig. $J$ Liposome Res 1989;1:211-22.

12 Taylor KMG, Taylor G, Kellaway IW, Stevens J. The influence of liposomal encapsulation on sodium cromoglycate pharmacokinetics in man. Pharmaceut Res 1989;6:633-6.

13 Debs RL, Straubinger RM, Brunette EN, Lin JM, Lin EJ, Montgomery $\mathrm{AB}$, et al. Selective enhancement of pentamidine uptake in the lung by aerosolization and delivery in liposomes. Am Rev Respir Dis 1987;135: 731-7.

14 Gilbert BE, Six HR, Wilson SZ, Wyde PR, Knight V. Small particle aerosols of enviroxime-containing liposomes. Antivir Res 1988;9:355-65.

15 Mihalko PJ, Schrier H, Abra RM. Liposomes: a pulmonary perspective. In: Gregoriadios G, ed. Liposomes as drug carriers. New York: Wiley, 1988: 679-94.

16 Shek PN, Jurima-Romet M, Barber RF, Demeester J. Liposomes: potential for inhalation prophylaxis and therapy. $J$ Aerosol Med 1988;1:257-8.

17 Meisner D, Pringle J, Mezei M. Liposomal pulmonary delivery I. In vivo disposition of atropine base in solution and liposomal form following endotracheal instillation to the rabbit lung. Journal of Microencapsulation 1989;6:379-87.

18 Taylor KMG, Taylor G, Kellaway KW, Stevens J. The stability of liposomes to nebulisation. Int J Pharmaceut 1990;58:57-61.

19 Barber RF, Shek PN. Liposome stability during ultrasonic nebulization [abstract]. In: Proceedings of the 33rd Harden Conference. London: Biochemical Society, 1989:53.

20 Radhakrish R, Mihalko PH, Abra RM. Method and apparatus for administering dehydrated liposomes by inhalation. 1990. (US patent No. 4895719.)

21 Farr SJ, Kellaway IW, Carman-Meakin B. Assessing the potential of aerosol-generated liposomes from pressurised pack formulations. Journal of Controlled Release 1987;5:119-27.

22 McGuirk JG, Ross AR, Gilroy GC. A novel method for the in-situ preparation of liposomes using an aerosol delivery system [abstract]. $J$ Pharmac Pharmacol 1986;38:20P.

23 Kirby C, Clarke J, Gregoriadis G. Effect of cholesterol content of small unilamellar liposomes on their stability in vivo and in vitro. Biochem $J$ 1980;186:591-8. 